
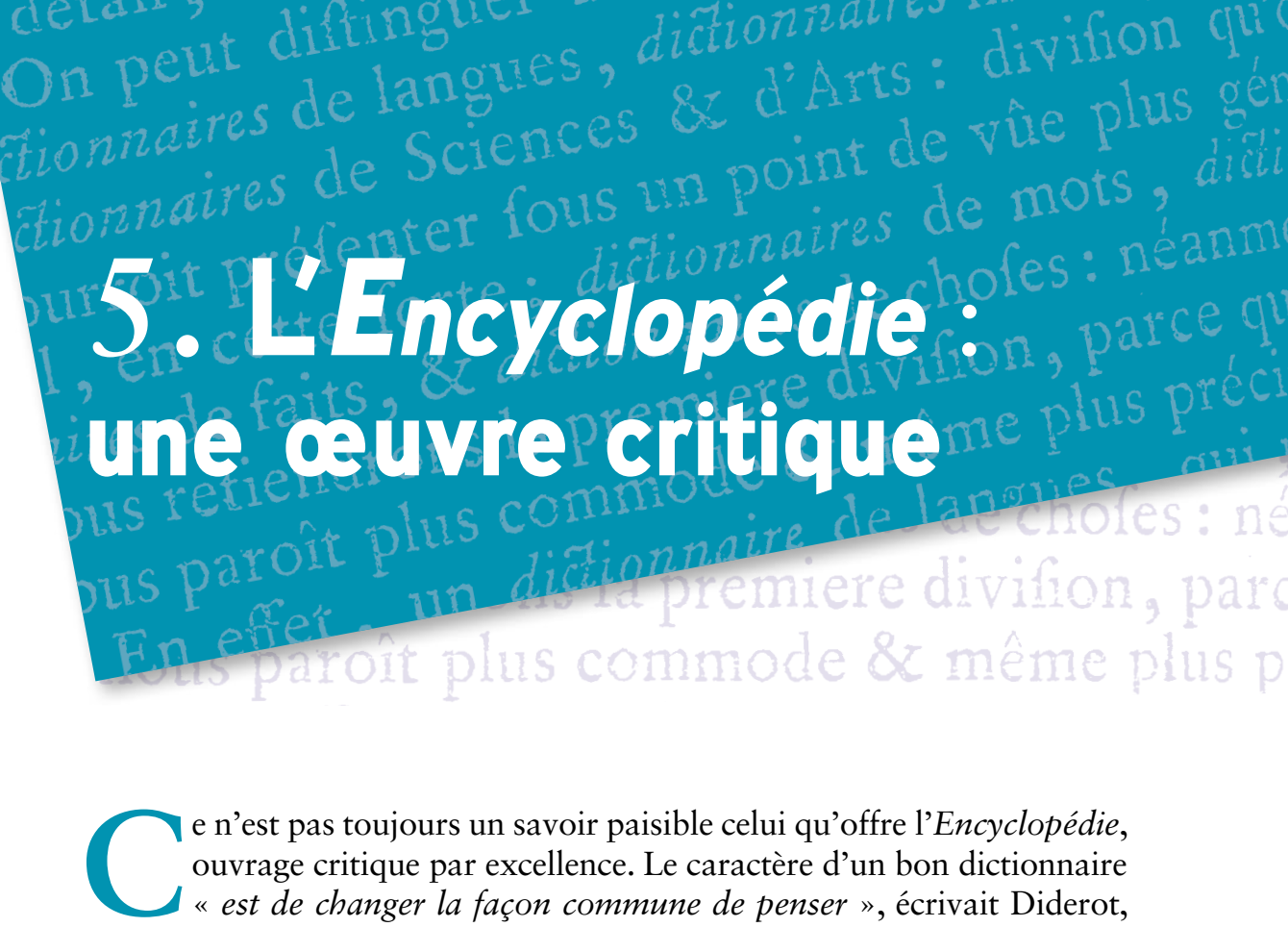

e n'est pas toujours un savoir paisible celui qu'offre l'Encyclopédie, ouvrage critique par excellence. Le caractère d'un bon dictionnaire " est de changer la façon commune de penser ", écrivait Diderot, et ces majestueux in-folio à l'aspect vénérable sont, de fait, traversés par les plus importants combats politiques, religieux, moraux, scientifiques du temps. Évoquons-en quelques-uns.

\title{
I. Critique politique
}

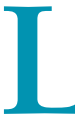

e combat politique direct est sans doute le plus subtilement exprimé, comme cela ne pouvait manquer dans un ouvrage publié, à ses débuts, avec autorisation royale. En régime de monarchie absolue et de droit divin, on n'en relève pas moins, à côté d'articles parfaitement conformes aux attentes des censeurs, des affirmations audacieuses sur les sources mêmes du pouvoir royal. Ainsi l'article Autorité POlITIQue, qui fit d'ailleurs scandale, commence-t-il par stipuler :

Aucun homme n'a reçu de la nature le droit de commander aux autres. [...] La puissance qui s'acquiert par la violence n'est qu'une usurpation [...]. La puissance qui vient du consentement des peuples suppose nécessairement des conditions qui en rendent l'usage légitime, utile à la société, avantageux à la république, qui la fixent et la restreignent entre des limites: car l'homme ne doit ni ne peut se donner entièrement et sans réserve à un autre homme. 
L'ouvrage étant surveillé de près par les censeurs, c'est donc souvent dans des articles d'apparence anodine que l'Encyclopédie fourmille d'exemples jugeant sévèrement la politique du gouvernement royal. Qui s'inquiéterait en effet du danger de l'article FaIM, AppétIT ? On y lit pourtant : "Lorsque le peuple meurt de faim, ce n'est jamais la faute de la Providence, c'est toujours celle de l'administration"; ou de l'article FrICHES, où on lit : "On peut mesurer sur l'étendue des friches dans un pays les progrès de la mauvaise administration, de la dépopulation et du mépris de l'agriculture "; ou encore de l'article Fouler : "On foule les peuples lorsqu'on les charge d'impôts excessifs ". Quant aux privilèges de naissance et aux prétentions nobiliaires, avec quelle dérision le chevalier de Jaucourt les traite-t-il : "Si l'on avait la généalogie exacte et vraie de chaque famille, il est plus que vraisemblable qu'aucun homme ne serait estimé ni méprisé à l'occasion de sa naissance " (art. GÉNÉALOGIE).

L'omniprésence de l'argent et de la corruption est dénoncée par Rousseau dans l'article Économie ainsi que "la mauvaise administration des deniers de l'État »:

la vénalité [est] poussée à tel excès que la considération se compte avec les pistoles, et que les vertus mêmes se vendent à prix d'argent : telles sont les causes les plus sensibles de l'opulence et de la misère, de l'intérêt particulier substitué à l'intérêt public, de la haine mutuelle des citoyens, de leur indifférence pour la cause commune, de la corruption du peuple, et de l'affaiblissement de tous les ressorts du gouvernement. [...] La patrie ne peut subsister sans la liberté, ni la liberté sans la vertu, ni la vertu sans les citoyens : vous aurez tout si vous formez des citoyens; sans cela vous n'aurez que de méchants esclaves, à commencer par les chefs de l'État. Or former des citoyens n'est pas l'affaire d'un jour ; et pour les avoir hommes, il faut les instruire enfants.

La dénonciation des privilèges est indissociable de celle des impôts iniques levés sur le peuple et dont noblesse et clergé sont exemptés. Voyez l'article TAILlE de Jaucourt : "Un malheureux journalier qui ne possède aucun fonds dans une paroisse, qui manque de travail, ne peut aller dans une autre où il trouve de quoi subsister sans payer la taille en deux endroits pendant deux

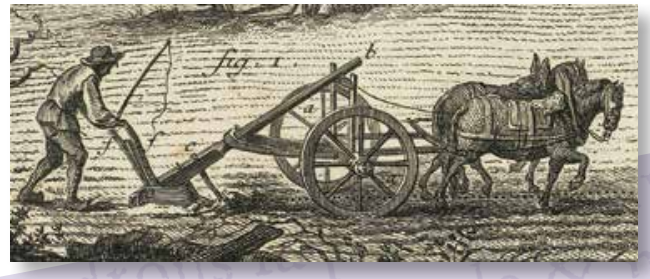
ans, et pendant trois s'il passe dans une troisième élection. "

Extrait de la vignette de la planche I de l'Agriculture (vol. I). 
Quant à la terrible gabelle :

La douleur s'empare de notre cœur à la lecture de l'ordonnance des gabelles. Une denrée que les faveurs de la providence entretiennent à vil prix pour une partie des citoyens, est vendue chèrement à tous les autres. Des hommes pauvres sont forcés d'acheter au poids de l'or une quantité marquée de cette denrée, et il leur est défendu, sous peine de la ruine totale de leur famille, d'en recevoir d'autres, même en pur don. (art. SEL impôt sur le, Jaucourt)

\section{Critique relligieuse}

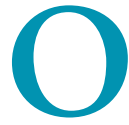

$\mathrm{n}$ trouve, dans l'Encyclopédie, des articles strictement orthodoxes et favorables aux papes et au clergé, mais aussi de nombreuses mises en cause des institutions catholiques, par exemple celle du célibat des prêtres. "Les ministres Protestants trouvent fort bien le temps d'avoir des enfants, de les élever, de gouverner leur famille, et de veiller sur leur paroisse. Ce serait offenser nos ecclésiastiques que de n'en pas présumer autant d'eux. " (art. CÉlibAT). Plus rarement, c'est le dogme même - ici celui de la résurrection des corps - qui est allusivement moqué : "Marcher, voir, entendre, parler, se mouvoir, quand on n'a plus ni pieds, ni mains, ni yeux, ni oreilles, ni organes actifs! Ceux qui sont morts le sont bien, et pour longtemps" (art. REvenANT)

Dans les années 1750, les encyclopédistes furent attaqués par l'ordre religieux le plus puissant du Royaume, celui des jésuites, qui, dans leur mensuel, menèrent ce qu'ils nommaient eux-mêmes « la guerre de l'Encyclopédie ». À l'époque, l'enseignement en France était majoritairement dispensé aux garçons par les jésuites, dans leurs collèges. D’Alembert conçoit donc un article Collège dans lequel il s'en prend, avec verve, aux fondements mêmes de l'enseignement jésuite, à savoir : la pure dévotion au latin, les "puérilités pédantesques de la rhétorique ", en particulier les exercices dits amplifications, "nom très convenable en effet, puisqu'ils consistent pour l'ordinaire à noyer dans deux feuilles de verbiage ce qu'on pourrait et ce qu'on devrait dire en deux lignes ". D'Alembert plaide pour un tout autre cursus : "Commencer par la philosophie, car il faut penser avant que d'écrire ». Il dénonce également la "corruption des mœurs ", entendons celle des jeunes garçons, de même que l'enseignement du préjugé nobiliaire : "On élève beaucoup de jeune noblesse; on leur parle à chaque instant de leur naissance et de leur grandeur, et par là on leur inspire, sans le vouloir, des sentiments d'orgueil à l'égard des autres. " Enfin, D’Alembert raille la frivolité des sujets et des méthodes 
d'enseignement, notamment la pratique des ballets, autrement dit la chorégraphie jésuite...

Quant aux jansénistes, secte catholique opposée aux jésuites «molinistes ", le même D'Alembert dénonce la violence de la "guerre insensée » qu'ils mènent entre eux :

La postérité aura-t-elle pour les auteurs de ces troubles de la pitié ou de l'indignation, quand elle saura qu'une dissension si acharnée se réduit à savoir si les cinq propositions expriment ou non la doctrine de l'évêque d'Ypres [Jansénius] ? Car tous s'accordent à condamner ces propositions en elles-mêmes. On appelle (très improprement) Jansénistes, ceux qui refusent de signer que Jansénius ait enseigné ces propositions. Ceux-ci de leur côté qualifient (non moins ridiculement) leurs adversaires de Molinistes, quoique le Molinisme n'ait rien de commun avec le formulaire ; et ils appellent athées les hommes sages qui rient de ces vaines contestations (art. FORMULAIRE).

Il s'attaque aussi à la croyance aux miracles de cette "secte de fanatiques " dans l'article Convulsionnaires.

"Fanatiques »! Sans doute est-ce un des plus acharnés qu'elle ait mené que le combat de l'Encyclopédie contre la barbarie aux mille visages, à commencer justement par le fanatisme et l'intolérance. Les massacres des guerres de religion demeurent encore dans toutes les mémoires et, plus encore, la Révocation de l'Édit de Nantes, en 1685, par Louis XIV sur le conseil des jésuites, qui avait déclenché les persécutions subies par les protestants, contraints de se convertir au catholicisme, et provoqué l'exil forcé de dizaine de milliers d'entre eux.

"Le mot intolérance s'entend communément de cette passion féroce qui porte à haïr et à persécuter ceux qui sont dans l'erreur. " Diderot poursuit :

L'intolérance ecclésiastique consiste à regarder comme fausse toute autre religion que celle que l'on professe et à le démontrer sur les toits, sans être arrêté par aucune terreur, par aucun respect humain. [...]

Tout moyen qui relâche les liens naturels et éloigne les pères des enfants, les frères des frères, les sœurs des sœurs, est impie.

Tout moyen qui tendrait à soulever les hommes, à armer les nations et tremper la terre de sang, est impie.

Il est impie de vouloir imposer des lois à la conscience, règle universelle des actions. Il faut l'éclairer et non la contraindre. (art. INTOLÉRANCE)

Pour prendre la mesure de l'audace d'un tel article, il faut savoir qu'à la même époque, dans le Dictionnaire de Trévoux, les jésuites revendiquaient 
l'intolérance en expliquant au mot "Intolérant » : "Il n'y a, à proprement parler, que les Catholiques dont les principes soient intolérants parce qu'il n'y a qu'eux qui ont les vrais principes".

L'auteur de l'article Tolérance de l'Encyclopédie, le pasteur Romilly, apostrophe les fanatiques : "Vous-mêmes qui me persécutez, pourriez-vous jamais vous résoudre à renier votre croyance? Ne feriez-vous pas aussi votre gloire de cette constance qui vous irrite et qui vous arme contre moi ? Pourquoi voulez-vous donc me forcer, par une inconséquence barbare, à mentir contre moi-même et à me rendre coupable d'une lâcheté qui vous ferait horreur? ". Quant à la Révocation de l'Édit de Nantes, si elle a trouvé des défenseurs chez certains ecclésiastiques encyclopédistes, comme l'abbé Mallet, elle est désignée par Diderot comme une faute majeure : "Louis XIV en persécutant les protestants, a privé son royaume de près d'un million d'hommes industrieux qu'il a sacrifiés aux vues intéressées et ambitieuses de quelques mauvais citoyens, qui sont les ennemis de toute liberté de penser, parce qu'ils ne peuvent régner qu'à l'ombre de l'ignorance » (art. RÉfUGIÉs). Et Voltaire ne manque pas de souligner que le fanatisme religieux a frappé l'Encyclopédie elle-même : «On a voulu priver le public de ce Dictionnaire utile, heureusement on n'y a pas réussi. Des âmes de boue, des fanatiques absurdes, préviennent tous les jours les puissants, les ignorants, contre les philosophes; si malheureusement on les écoutait, nous retomberions dans la barbarie dont les seuls philosophes nous ont tirés. » (art. Heureux, Heureuse, Heureusement).

FANATISME enfin, donne lieu à un long article qui analyse le phénomène à travers l'histoire :

Parcourez tous les ravages de ce fléau, sous les étendards du croissant, et voyez dès les commencements, un Calife assurer l'empire de l'ignorance et de la superstition en brûlant tous les livres. [...] Bientôt un autre Calife contraindra les Chrétiens à la circoncision, tandis qu'un empereur chrétien force les Juifs à recevoir le baptême.

Deleyre, auteur de l'article, poursuit :

On ne sait guère quel parti prendre avec un corps de fanatiques : ménagez-les, ils vous foulent aux pieds; si vous les persécutez, ils se soulèvent. [...] II n'y a que le mépris et le ridicule qui puissent les décréditer et les affaiblir. On dit qu'un chef de police, pour faire cesser les prestiges du fanatisme, avait résolu, de concert avec un chimiste célèbre, de les faire parodier à la foire par des charlatans.

Conseil encyclopédique à méditer de nos jours... 
L'article JUIF, de Jaucourt, rappelle " combien, en tout lieu, on s'est joué de cette nation d'un siècle à l'autre. On a confisqué leurs biens, lorsqu'ils recevaient le Christianisme; et bientôt après on les a fait brûler, lorsqu'ils ne voulurent pas le recevoir ». Et Jaucourt, citant Montesquieu, avertit son propre siècle : "Si quelqu'un dans la postérité ose dire qu'au dix-huitième siècle tous les peuples de l'Europe étaient policés, on citera l'inquisition pour prouver qu'ils étaient en grande partie des barbares" (art. INQUISITION).

\section{Dénonciation des barbaries}

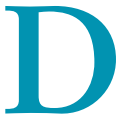

autres manifestations de barbarie hantent le XVIII ${ }^{\mathrm{e}}$ siècle, à commencer par celle de l'esclavage. On trouve certes un article écrit par un " colon " qui traite du " commerce des nègres ", mais l'Encyclopédie contient aussi le premier texte ouvertement abolitionniste publié en France, l'article TRAITE DES NÈGRES de Jaucourt : "Cet achat de nègres, pour les réduire en esclavage, est un négoce qui viole la religion, la morale, les lois naturelles, et tous les droits de la nature humaine ", et qui tient des "usages arbitraires et inhumains des colonies".

On dira peut-être qu'elles seraient bientôt ruinées ces colonies, si l'on y abolissait l'esclavage des nègres. [...] Il est vrai que les bourses des voleurs de grand chemin seraient vides, si le vol était absolument supprimé ; mais les hommes ont-ils le droit de s'enrichir par des voies cruelles et criminelles ? [...] Non... Que les colonies européennes soient donc plutôt détruites, que de faire tant de malheureux!

Barbarie aussi, celle de la torture, dite "question ". Si un juriste y explique froidement les différents procédés utilisés par les bourreaux pour obtenir l'aveu, Jaucourt, lui, met en accusation la pratique elle-même :

La loi de la nature crie contre cette pratique, sans y mettre aucune exception vis-à-vis de qui que ce soit. Indépendamment de la voix de l'humanité, la question ne remplit point le but auquel elle est destinée. Que dis-je, c'est une invention sûre pour perdre un innocent qui a la complexion faible et délicate, et sauver un coupable qui est né robuste. Ceux qui peuvent supporter ce supplice, et ceux qui n'ont pas assez de force pour le soutenir, mentent également. Le tourment qu'on fait souffrir dans la question est certain, et le crime de l'homme qui souffre ne l'est pas; ce malheureux que vous appliquez à la torture songe bien moins à déclarer ce qu'il sait qu'à se délivrer de ce qu'il sent. (art. QUESTION) 


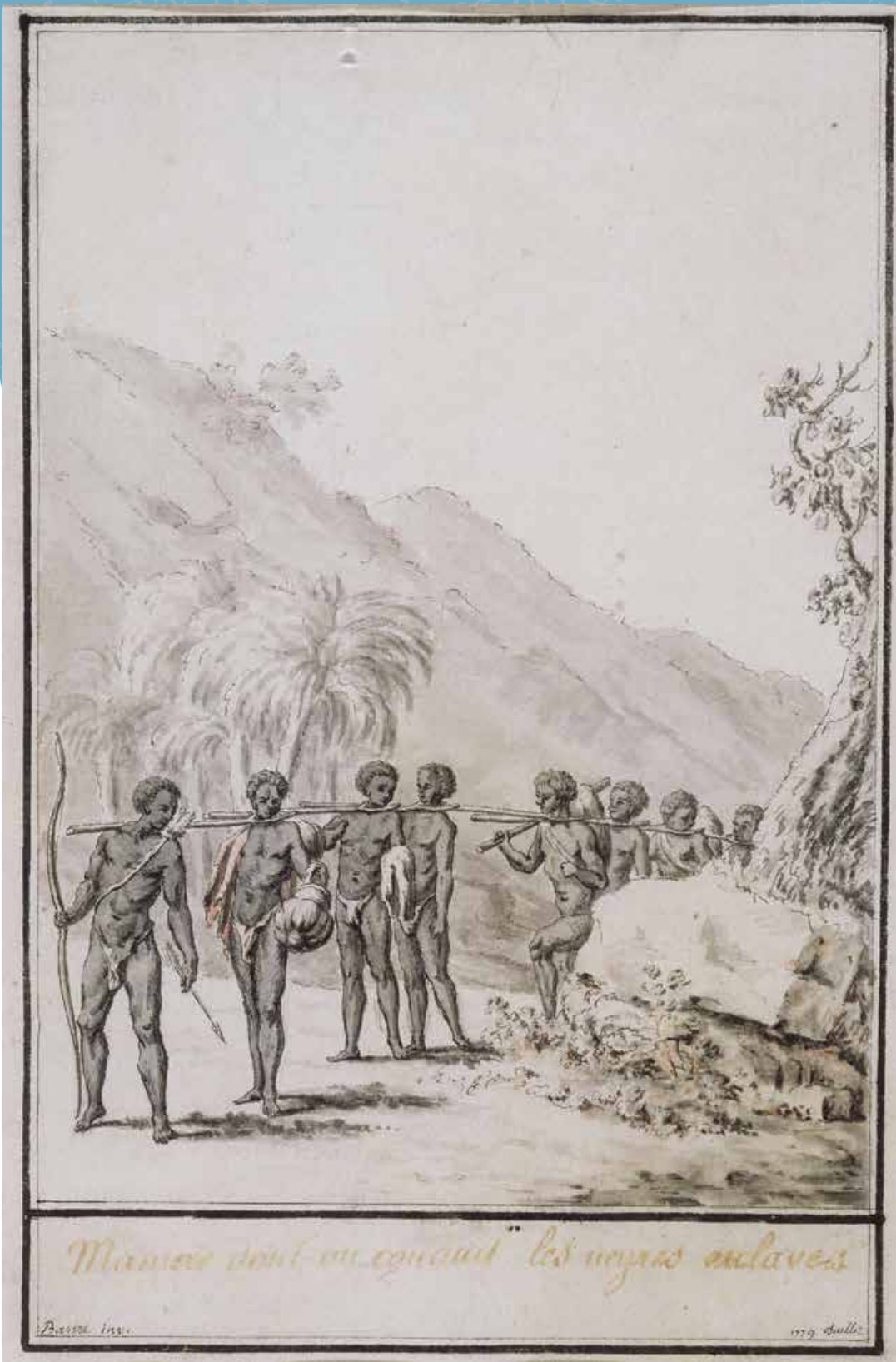

«Manière dont on conduit les nègres esclaves » (gravure de James Basire). 


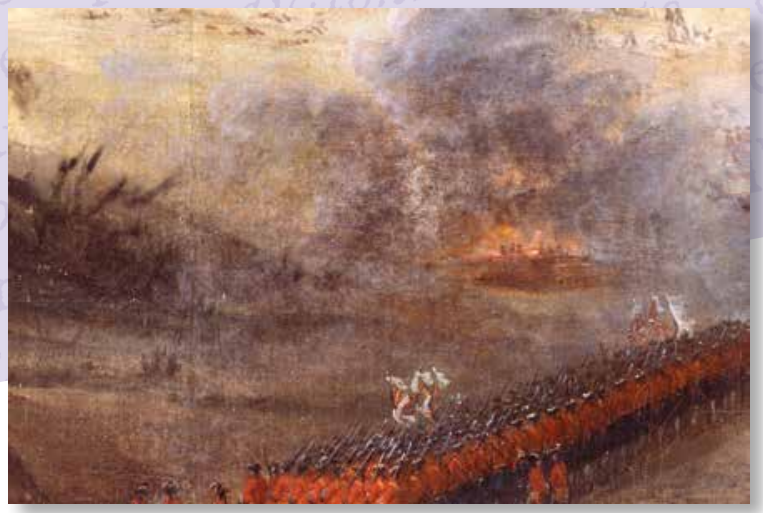

Détail de La Bataille de Fontenoy le 11 mai 1745 , tableau de Pierre Lenfant (Paris, Musée de l'Armée).

Quant à l'entreprise guerrière, Jaucourt la dénonce avec la même fermeté : "La guerre étouffe la voix de la nature, de la justice, de la religion, et de l'humanité. Elle n'enfante que des brigandages et des crimes; avec elle marche l'effroi, la famine, et la désolation; elle déchire l'âme des mères, des épouses et des enfants; elle ravage les campagnes, dépeuple les provinces, et réduit les villes en poudre. » (art. GUERRE)

Et il ne s'agit pas seulement de proclamations d'indignation : c'est de situations bien concrètes, celle du sort des soldats, par exemple, dont certains encyclopédistes se préoccupent : Saint-Lambert, poète et officier de l'armée royale, présente, dans son article TRANSFUGE, un plaidoyer nourri et argumenté contre l'enrôlement de force et la peine de mort qui, en France, punissait les soldats qui désertaient par milliers.

Ce sont ces hommes plutôt enchaînés qu'engagés, qu'on punit de mort lorsqu'ils veulent rompre des chaînes qui leur pèsent. [...] Quelles ont été jusqu'à présent les suites de vos arrêts sanguinaires et de tant d'exécutions? Depuis que les déserteurs sont punis de mort en France, y en a-t-il moins qu'il y en avait autrefois ? [...] non [...], la désertion est aussi commune dans vos troupes qu'elle l'était auparavant.

\section{Combats pour une autre morale}

Changer la façon commune de penser " implique bien sûr de nouvelles valeurs morales, fondées sur l'humanité, la bienveillance, la recherche du bonheur; ainsi Diderot se préoccupe-t-il, très différemment des jésuites, de l'éducation des enfants, notamment de l'éveil de 
leur sensibilité à la justice: "Malheur aux enfants qui n'auront jamais vu couler les larmes de leurs parents au récit d'une action généreuse: malheur aux enfants qui n'auront jamais vu couler les larmes de leurs parents sur la misère des autres." (art. LocKe, PHILOSOPHIE DE).

En opposition à l'austérité et au goût du malheur que professent certains moralistes chrétiens, Diderot s'exclame :

Ceux qui enseignent je ne sais quelle doctrine austère qui nous affligerait [...] sur cette foule d'objets qui nous entourent et qui sont destinés à émouvoir cette sensibilité en cent manières agréables, sont des atrabilaires à enfermer aux petites-maisons [l'hôpital pour les fous]. Ils remercieraient volontiers l'être tout-puissant d'avoir fait des ronces, des épines, des venins, des tigres, des serpents, en un mot tout ce qu'il y a de nuisible et de malfaisant; et ils sont tout prêts à lui reprocher l'ombre, les eaux fraîches, les fruits exquis, les vins délicieux, en un mot les marques de bonté et de bienfaisance qu'il a semées entre les choses. (art. VoluptUeuX)

S'étonnera-t-on, dès lors, de trouver dans ce dictionnaire des sciences et des métiers un éloge de l'amour et du plaisir amoureux ? On ira lire l'article MARIAGE (Médecine et Diète) du médecin Ménuret de Chambaud et, d'abord, l'évocateur article Jouissance de Diderot :

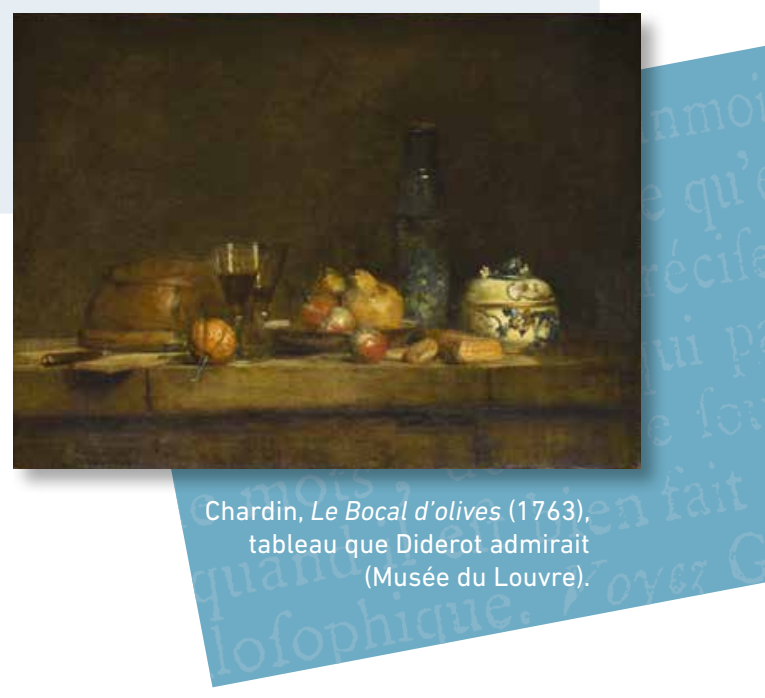

S'il y avait quelque homme pervers qui pût s'offenser de l'éloge que je fais de la plus auguste et la plus générale des passions, j'évoquerais devant lui la Nature, je la ferais parler, et elle lui dirait. Pourquoi rougis-tu d'entendre prononcer le nom d'une volupté dont tu ne rougis pas d'éprouver l'attrait dans l'ombre de la nuit ? Ignores-tu quel est son but et ce que tu lui dois? Crois-tu que ta mère eût exposé sa vie pour te la donner si je n'avais pas attaché un charme inexprimable aux embrassements de son époux? Tais-toi, malheureux, et songe que c'est le plaisir qui t'a tiré du néant. 


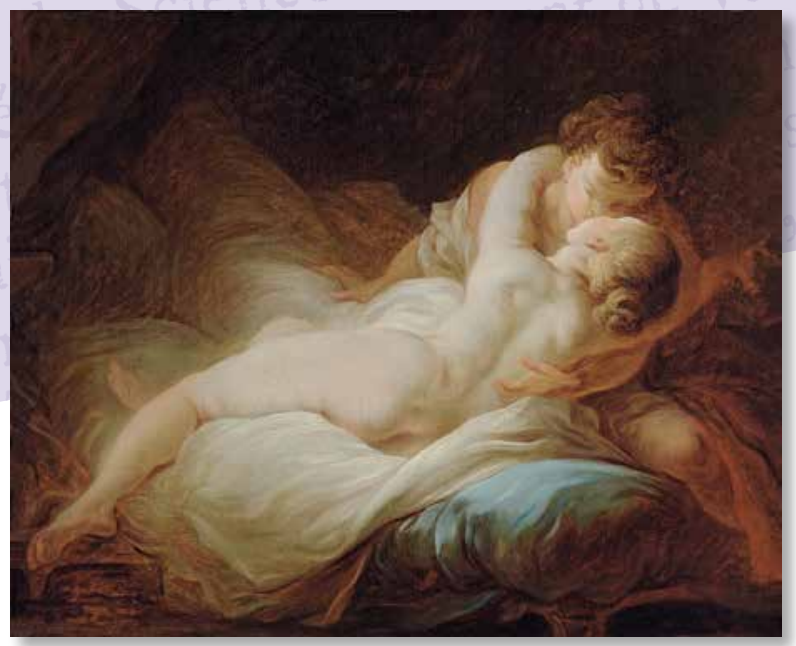

Fragonard,

L'Instant désiré

(coll. part.).

\section{Combats contre les interdits de pensée}

T a reconnaissance des découvertes scientifiques passe par le combat contre les interdits de pensée. D’Alembert, dans le "Discours préliminaire des éditeurs ", dénonce "le despotisme théologique " dans l'histoire : "l'abus de l'autorité spirituelle réunie à la temporelle

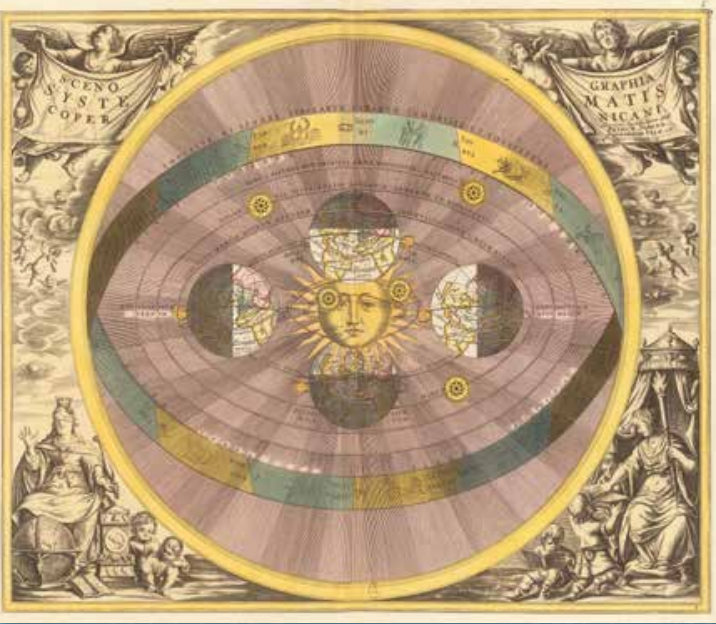

D'Alembert milite pour la levée des interdictions qui pèsent sur l'enseignement du système de Copernic, mis à l'index (estampe aquarellée du XvII siècle représentant ce système, BnF). 
forçait la raison au silence; et peu s'en fallut qu'on ne défendît au genre humain de penser". Ainsi dans l'article AnTIPODEs, D'Alembert rappelle avec ironie qu'un pape déclara hérétique un prêtre qui avait émis l'idée qu'il existait des hommes aux antipodes. Ailleurs il énumère les persécutions subies par les savants : «En Italie, pays d'inquisition, il est défendu de soutenir le système de Copernic, qu'on regarde comme contraire à l'Écriture à cause du mouvement de la Terre que ce système suppose "; quant au "grand Galilée », il "fut autrefois mis à l'inquisition, et son opinion du mouvement de la Terre condamnée comme hérétique. "(art. COPERNIC)

Les articles de mathématiques peuvent aussi servir à dégager la pensée des cadres répétitifs de l'apprentissage scolastique et à préparer un peuple à s'ouvrir à l'esprit philosophique. Ainsi lit-on dans l'article GÉOMÈTRE de D'Alembert :

Mais indépendamment des usages physiques et palpables de la Géométrie, nous envisagerons ici ses avantages sous une autre face, à laquelle on n'a peut-être pas fait encore assez d'attention : c'est l'utilité dont cette étude peut être pour préparer comme insensiblement les voies à l'esprit philosophique, et pour disposer toute une nation à recevoir la lumière que cet esprit peut y répandre. C'est peut-être le seul moyen de faire secouer peu à peu à certaines contrées de l'Europe, le joug de l'oppression et de l'ignorance profonde sous laquelle elles gémissent. Le petit nombre d'hommes éclairés qui habitent certains pays d'inquisition se plaint amèrement, quoiqu'en secret, du peu de progrès que les Sciences ont fait jusqu'ici dans ces tristes climats. Les précautions qu'on a prises pour empêcher la lumière d'y pénétrer ont si bien réussi que la Philosophie y est à peu près dans le même état où elle était parmi nous du temps de Louis le Jeune.

Autre combat de grande ampleur que répercute l'Encyclopédie, celui qui milite pour l'inoculation contre la variole. D'importants théologiens la condamnaient comme pratique hérétique qu'il fallait interdire puisque c'était "usurper les droits de la Divinité, que de donner une maladie à celui qui ne l'a pas, ou d'entreprendre d'y soustraire celui qui, dans l'ordre de la Providence, y était naturellement destiné ", comme le rapporte l'académicien des sciences et encyclopédiste La Condamine - qui lança une campagne pour l'inoculation en 1754 et dont l'Encyclopédie reproduit les arguments dans une première partie de l'article InOCUlation ; Voltaire se joignit à cette campagne, ainsi que l'Encyclopédie sous les auspices de Tronchin (voir le chapitre 4), suscitant une véritable mobilisation de l'opinion publique. 


\section{Critique des savoirs et de leur transmission}

$\mathrm{L}$

Encyclopédie, loin de se limiter à accumuler les connaissances, est aussi un lieu de critique des savoirs et de leur diffusion. Cela passe d'abord par le choix des connaissances que contient l'Encyclopédie et la décision d'en exclure tout ce qui ne sert pas une transmission utile à la science et à la vertu - car l'une des préoccupations fondamentales des encyclopédistes a été de distinguer ce qui relève des talents et ce qui relève des titres de noblesse, et d'illustrer le mérite plutôt que la naissance. C'est ainsi que D'Alembert explique :

On ne trouvera donc dans cet ouvrage [...] ni la généalogie des grandes Maisons, mais la généalogie des Sciences, plus précieuse pour qui sait penser ; [...] ni les Conquérants qui ont désolé la terre, mais les génies immortels qui l'ont éclairée; ni enfin une foule de Souverains que l'Histoire aurait dû proscrire. Le nom même des Princes et des Grands n'a droit de se trouver dans l'Encyclopédie que par le bien qu'ils ont fait aux Sciences ; parce que l'Encyclopédie doit tout aux talents, rien aux titres, et qu'elle est l'histoire de l'esprit humain, et non de la vanité des hommes. (« Avertissement » du volume III).

Mais les connaissances elles-mêmes, telles qu'elles se forment et se diffusent, sont également soumises à un examen critique. Par exemple, dans Musique, article de tête de sa contribution entièrement rédigée en 1749, Rousseau discute notamment des mérites comparés de la musique de son temps et de la musique des anciens : il tend à relativiser le point de vue moderne qui postule un progrès de l'une à l'autre. Dans un paragraphe lié aux planches, il élargit encore ce point de vue relativiste en introduisant trois références à des musiques extra-européennes (voir illustration). Toutefois, les exemples qu'il présente, tirés de sources livresques françaises, sembleraient plutôt démontrer le contraire. D'où la réflexion critique de Rousseau qui - comme il le fera quelques années plus tard dans son Discours sur l'origine de l'inégalité à propos des récits de voyage et de la valeur de leurs observations - questionne le processus même de transmission d'une donnée exotique :

On a ajouté, dans la même planche, un air chinois tiré du père du Halde ; et, dans une autre planche, un air persan tiré du chevalier Chardin ; et ailleurs, deux chansons des sauvages de l'Amérique, tirées 

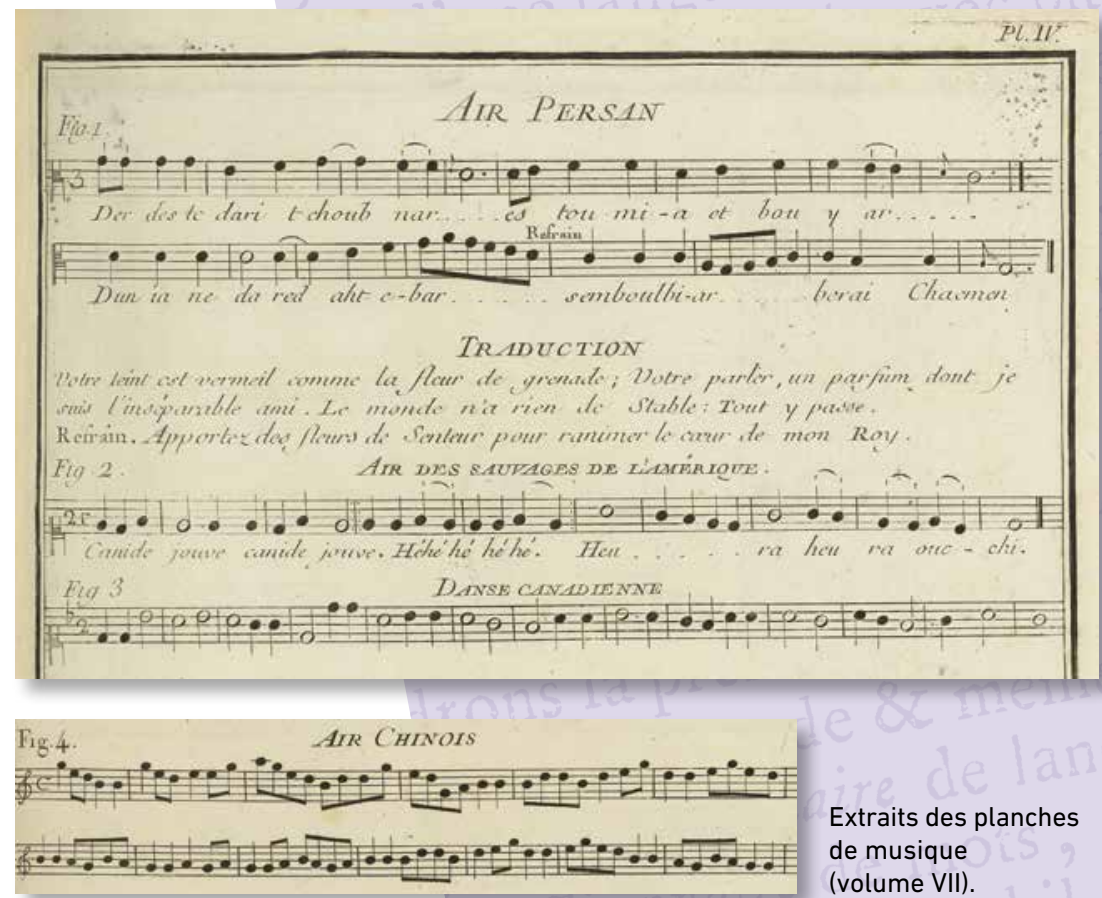

Extraits des planches de musique (volume VII).

du père Mersenne. On trouvera dans tous ces morceaux une conformité de modulation avec notre musique, qui pourra faire admirer aux uns la bonté et l'universalité de nos règles, et peut-être rendre suspecte à d'autres la fidélité ou l'intelligence de ceux qui ont transmis ces airs.

Les connaissances consignées dans l'Encyclopédie proviennent parfois d'ouvrages de compilation, et donc de récits plus ou moins fiables de voyageurs lointains. D'où, chez Diderot, une critique ironique des sources du savoir, qui sont autant d'appels à douter de leur validité :

${ }^{*}$ AguAPA, s. m. (Hist. nat. bot.), arbre qui croît aux Indes occidentales, dont on dit que l'ombre fait mourir ceux qui s'y endorment nus, et qu'elle fait enfler les autres d'une manière prodigieuse. Si les habitants du pays ne le connaissent pas mieux qu'il ne nous est désigné par cette description, ils sont en grand danger. 
* Acacalis, s. m., arbrisseau qui porte une fleur en papillon, et un fruit couvert d'une cosse. Voyez Ray. Hist. Plant. On lit dans Dioscoride [...]. Malgré toutes ces autorités, je ne regarde pas le sort de l'acacalis comme bien décidé ; sa description est trop vague, et il faut attendre ce que les progrès de l'histoire naturelle nous apprendront là-dessus.

À la recherche des mots aguapa ou acacalis, le lecteur aura appris ici non ce que sont ces plantes lointaines et improbables, mais une forme supérieure de la critique : questionner ses questionnements, et même parfois en rire !

"Il faut attendre "... Cette formule ne cesse de revenir dans le dictionnaire, comme un appel à la vigilance. Pour les encyclopédistes, l'inlassable recherche du savoir est inséparable d'une conscience : celle de la précarité des connaissances ou, plus exactement, de leur perpétuelle caducité et de leur éternel renouvellement. 